\title{
Klinische Studien bei Kindern - Besonderheiten, Herausforderungen, neue Modelle zur operativen Durchführung
}

10 Jahre nach Inkrafttreten der EU Verordnung ${ }^{1}$ zur Förderung der Entwicklung von Kinderarzneimitteln in Europa ist die Anzahl der pädiatrischen Entwicklungspläne (EU Paediatric Investigation Plans [PIP]), der daraus resultierenden klinischen Studien und der in diese Studien einzuschließenden Kinder stetig gestiegen².

Schon während der Planung des pädiatrischen Programms werden die Weichen für eine erfolgreiche operative Implementierung und Durchführung gestellt. Da die Planung, gemäß EU-Verordnung, schon früh im Entwicklungsprozess eines Arzneimittels erfolgen soll, fehlen dann oft noch entscheidende Informationen. Außerdem orientieren sich die pädiatrischen Indikationen häufig an der geplanten Indikation in Erwachsenen, was zu einer Häufung an pädiatrischen Entwicklungsprogrammen in einzelnen, oft für Kinder gar nicht so relevanten Indikationen führt. Ein Ausweg wäre eine früh startende breite und mit zunehmender Verfügbarkeit neuer Informationen iterative wissenschaftliche Diskussion der geplanten Indikationen und des entsprechend angemessenen Studienprogramms mit Beteiligung von verantwortlichen Behörden, akademischen Experten, Patienten und forschenden Arzneimittelunternehmen.

Innovative Methoden zur Planung (z.B. adaptive Studiendesigns, Extrapolationsmodelle) und Durchführung (z. B. elektronische Einverständniserklärung, mobile Datenerhebung) werden noch nicht durchgängig angewendet. Damit wird eine Chance verpasst, die Studienplanung und Durchführung zu optimieren. Dies liegt vielfach an der Unsicherheit, ob, wann und wie diese Methoden richtig eingesetzt werden können. Hilfreich wäre hier eine offene Diskussion aller Beteiligten zum wissenschaftlichen Nutzen und

1 Paediatric Regulations (EC) No 1901/2006 of the European Parliament and of the Council on Medicinal Products for Paediatric Use

2 10-year Report to the European Commission EMA/231225/2015 zum sinnvollen Einsatz dieser neuen Methoden und hieraus resultierende Empfehlungen.

Der Aufbau der benötigten Studien-Infrastruktur (Ausstattung der Prüfzentren, Vorhandensein geschulter Studienärzte und -schwestern mit ausreichender Zeit für klinische Forschung) in Deutschland und in anderen europäischen Ländern konnte mit der sich vergrößernden Anzahl von klinischen Prüfungen für Kinder nicht in allen Bereichen schritthalten. Dies führt zu vielfältigen Herausforderungen bei der Planung und operativen Durchführung von Arzneimittelstudien in Kindern und zu einer steigenden Konkurrenz um die knappen Ressourcen zwischen akademischen Studien und Industrie-finanzierten Studien zur Entwicklung neuer Arzneimittel für Kinder. Hinzu kommt, dass aufgrund der vergleichsweise geringen Anzahl an Kindern, die pro Zentrum/pro Land an einer Studie teilnehmen, in der Regel deutlich mehr Prüfzentren als bei Studien mit einer vergleichbaren Anzahl von Erwachsenen benötigt werden.

Besonderer Wert muss auch auf die Information und Einverständniserklärung für Studienvorhaben gelegt werden, die für Kinder und Eltern verständlich sein muss. Art und Umfang der Aufklärung der kleinen Patienten und ihrer Eltern über die klinische Studie benötigen speziell geschultes Personal und mehr Zeit als bei erwachsenen Patienten. Um diese Prozesse zu verbessern und die Akzeptanz der Studie bei allen Beteiligten zu erhöhen, ist die frühe Einbeziehung von Patienten- und Elterninitiativen in die Planung der Studien und die Erstellung der Materialien für Patienten sehr wichtig. Eine Folge all dieser Herausforderungen ist, dass die klinischen Studien mit Kindern in der Regel mehr Zeit und Ressourcen benötigen als Studien mit Erwachsenen in einer vergleichbaren Indikation. So zeigen Vergleichsdaten, dass z. B. die Initiierung einer pädiatrischen Studie rund 6-8 Monate länger dauert als die Initiierung einer vergleichbaren Studie mit Erwachsenen ${ }^{3}$.
Viele dieser Herausforderungen können nur schwer von einem einzelnen Prüfzentrum oder einem einzelnen Unternehmen alleine dauerhaft adressiert werden. Daher haben sich in den letzten Jahren verschiedene internationale Initiativen gebildet, deren Ziel es ist, neue Methoden für pädiatrische Studien zu entwickeln und über die Bildung von pädiatrischen Studiennetzwerken den Aufbau von entsprechender Infrastruktur europaweit, aber auch in Deutschland, zu fördern und deren Erhalt dauerhaft zu sichern. Beispiele für solche Initiativen finden sich unter anderem unter dem Dach der „Innovative Medicines Initiative“ (IMI2), einer gemeinnützigen Kooperation zwischen der Europäischen Kommission (EC) und dem Europäischen Verband der Pharmazeutischen Industrie (EFPIA).

Neben einer Reihe von Projekten, die sich mit der Entwicklung neuer Methoden zur Identifizierung und frühen, präklinischen Prüfung von Medikamenten im Bereich pädiatrischer Tumoren befassen, ist das Ziel des zur Zeit größten IMI2-finanzierten Projekts in diesem Bereich die Verbesserung der Durchführung von pädiatrischen Studien und der nachhaltige Aufbau entsprechender Infrastruktur.

„conect4children“ (c4c) ${ }^{4}$ hat zum Ziel, ein europaweites, indikationsunabhängiges Netzwerk zur Planung und Durchführung pädiatrischer Studien zu schaffen. Dabei soll durch den Aufbau einer effektiven Netzwerkstruktur und einer frühen wissenschaftlichen Diskussion der Studienkonzepte sowohl die wissenschaftliche Aussagekraft als auch die Durchführbarkeit der Studien verbessert werden. Patienten- und Elternvertreter werden in alle

3 KMR Clinical Trial Cycle Time Metrix June 2017, Nordic Pediatric Conference, Helsinki 2017

4 Conect4children: Funded by the Innovative Medicines Initiative 2 Joint Undertaking under grant agreement No 777389. The Joint Undertaking receives support from the European Union's Horizon 2020 research and innovation programme and EFPIA. 
Aktivitäten eng eingebunden. An c4c beteiligen sich europaweit über 40 Partner (bestehende Netzwerken, Kliniken, Patientenorganisationen und Unternehmen). In Deutschland hat sich unter Leitung von Herrn Prof. Dr. Schwab (RBMF/IKP, Stuttgart $)^{5}$ eine Gruppe von ausgewählten Kli-

5 Robert Bosch Gesellschaft für Medizinische Forschung MBH, Dr. Margarete FischerBosch-Institut für klinische Pharmakologie, Stuttgart niken und Studienzentralen mit zusätzlichen Experten für Biometrie und ethische Fragen zusammengeschlossen, um eine entsprechende Struktur in Deutschland aufzubauen.

Interessenkonflikte

Die Autorin ist Mitarbeiterin der Bayer AG im Bereich Pädiatrische Entwicklung und Mitglied der Projektleitung „conect4children“.
Autorin

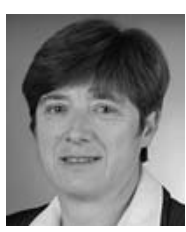

Heidrun Hildebrand Bayer AG, Pediatric Development

Korrespondenzadresse

\section{Heidrun Hildebrand}

Bayer AG

Research \& Development, Pharmaceuticals Pediatric Development

Building P300, A433

13342 Berlin

Germany

heidrun.hildebrand@bayer.com 